\title{
traitements non chirurgicaux des sialo-lithiases
}

RÉSUMÉ Objectifs : les lithiases salivaires représentent une pathologie bénigne des glandes salivaires. Les traitements par sialo-endoscopie et lithotripsie intra ou extra-corporelle ont été développés dans le monde comme alternative à la chirurgie. Notre objectif de départ a été de mettre au point des traitements non chirurgicaux afin de réduire au minimum la morbidité de cette pathologie. Dans cette étude, nous analysons une grande série clinique afin de déterminer le succès de ces nouvelles techniques mais aussi de développer des protocoles de traitement en fonction des pathologies.

But de l'étude : étudier rétrospectivement 1973 glandes salivaires présentant une pathologie et traitées non chirurgicalement.

Méthodes : traitement des lithiases salivaires utilisant soit l'endoscopie avec instrumentation endocanalaire, soit la lithotripsie intracanalaire par laser, soit la lithotripsie par ondes de chocs électromagnétiques sur 1973 glandes lithiasiques entre 1988 et 2004. $\mathrm{Au}$ total, 1205 endoscopies ont été pratiquées sur des glandes pathologiques et 768 lithotripsies extracorporelles ont été réalisées. Résultats : l'endoscopie a permis d'éliminer 96\% des lithiases examinées. La lithotripsie, quant à elle, a détruit complètement $63 \%$ des lithiases dont $35 \%$ de cas ont été totalement évacués, soit de façon spontanée soit en utilisant l'endoscopie.

Il n'y a jamais eu de complications majeures au niveau des nerfs facial ou lingual, aucun dommage dentaire n'a été relevé. Un patient au début de l'étude sur l'endoscopie a dû subir une intervention chirurgicale par blocage de la sonde à panier miniaturisée

Philippe KATZ

Institut d'Explorations Fonctionnelles des Glandes Salivaires,

7, rue Théodore de Banville, 75017 Paris. dans le bassinet de la glande. Les effets secondaires comme les infections, douleurs, œdèmes ou pétéchies ont été traités simplement sans conséquence pour les glandes salivaires. $4 \%$ des endoscopies et $2 \%$ des lithotripsies ont cependant en dernier recours nécessité l'ablation chirurgicale des glandes.

Conclusions : le traitement des lithiases salivaires ne doit plus aujourd'hui faire appel au seul traitement chirurgical, mais doit prendre en compte les nouvelles techniques d'endoscopie et la lithotripsie extra-corporelle. Ces deux nouvelles techniques sont particulièrement intéressantes pour les calculs situés dans les canaux salivaires et les bassinets des glandes. Cette étude démontre l'efficacité des nouveaux traitements.

\section{MOTS CLÉS}

sialolithiases

endoscopie

lithotripsie 


\section{introduction}

A

pproximativement $1,3 \%$ de la population présente un ou plus calcul salivaire se répartissant de la façon suivante :

- $76 \%$ dans la glande sub-mandibulaire ;

- $22 \%$ dans la glande parotide ;

$-2 \%$ dans la glande sub-linguale.

L'âge d'apparition des symptômes varie de 3 à 85 ans soit une moyenne de 44 ans[1].

Avant cette étude, seuls les traitements chirurgicaux étaient utilisés. La chirurgie consistait soit en l'ablation complète de la glande par voie transcutanée, soit en une dissection des canaux excréteurs par voie endobuccale à la recherche des lithiases. Cette chirurgie n'était pas sans risque pour les structures avoisinantes telles que le nerf facial pour la glande parotide ou le nerf lingual pour la glande submandibulaire. Le développement de l'endoscopie avec l'instrumentation endocanalaire et la lithotripsie extracorporelle ont permis une nouvelle alternative à la chirurgie conventionnelle.

\section{revue de la littérature}

Durant les 15 dernières années, simultanément l'endoscopie des glandes salivaires et la lithotripsie des lithiases ont été développées par de nombreuses équipes dans le monde[1].

- En 1986, Marmary et en 1989, Brouns ont décrit les premières expériences in vitro de fragmentation des calculs salivaires par l'intermédiaire de lithotripteurs rénaux[2, 3].

- En 1990 et 1991, Katz publia son expérience sur l'endoscopie des glandes salivaires à l'aide d'un endoscope ultrafin flexible. Il réalisa la première mondiale en $1988[4,5]$.

- Sterenborg rapporta son expérience in vitro de lithotripsie par laser en 1990[6]. Il compara l'effet du Dye Laser Pulsé (LFDL/3, Candela Corp., Wyland, Mass., USA) au laser HoYSGG (Laser 1-2-3, Schwartz Electro-
Optics, Inc., Orlando, Fl., USA). Ces lasers étaient performants pour fragmenter les lithiases, mais entraînaient des plaies au niveau des canaux salivaires. Onze calculs furent analysés pendant cette étude et l'on trouva que la composition était faite d'hydroxyapatite mélangé à d'autres sels minéraux.

- Grundlach, et al. rapportèrent leur expérience in vitro et sur 12 patients traités par endoscopie et lithotripsie par laser en 1990[7]. 18 calculs salivaires furent ainsi étudiés in vitro par laser et photoabsorption. Ils étudièrent les lasers $\mathrm{CO} 2, \mathrm{Nd}$ : YAG et XeCI Eximer et jugèrent que seul le laser Eximer était valable pour la fragmentation des sialolithiases.

- Konigsberger, et al. [8, 9] décrivirent leur première fragmentation d'un calcul 
salivaire sur un patient à l'aide d'un laser Eximer XeCI (Technolas CO.Munich, Germany) en 1990. En 1993, ils rapportèrent leur expérience sur une série de patients, utilisant un endoscope rigide et un lithotrypteur à onde de choc électro-hydraulique. Dans leur série, 19 des 23 patients eurent une fragmentation complète de leur calcul sans aucune complication. La sonde de fragmentation était placée à environ $1 \mathrm{~mm}$ devant le calcul et ils utilisaient une énergie de $150 \mathrm{~mJ}$ à une fréquence de $10 \mathrm{~Hz}$.

- Kater[10] décrivit ses trois années d'expérience utilisant un lithotrypteur à ondes de choc électromagnétiques (Minilith SL-1 ${ }^{\circledR} / \mathrm{K}$. Storz, Tuttlingen, Germany) en 1993.

- Toujours en 1993, Katz[11] rapporta son succès sur une série de patients traités par ablation des lithiases salivaires par endoscopie et sonde à panier miniaturisée.

- En 1995, Iro et Zenk[12] rapportèrent l'utilisation d'un Dye Laser Rhodamine 6-G pour la fragmentation des lithiases salivaires. Ils transposèrent leur expérience en 1989, de fragmentation in vitro qui avait montré un taux de réussite de $75 \%$ à l'homme qui donna des résultats de destruction complète pour $10 \%$ et partielle pour $55 \%$. Ils mirent en garde les praticiens voulant utiliser cette méthode contre les dommages sur les tissus avoisinants et les canaux salivaires dus au Laser Eximer utilisé pour la lithotripsie.

- En 1996, Arzoz, et al.[13] décrirent une série de 18 patients présentant des sialolithiases traitées par lithotripsie endoscopique, technique découlant de leur premier essai en 1994. Ils comparèrent le Lasertryp (Dornier Laser Impact Lithotryptor/Germering, Allemagne) à l'énergie pneumoblastique (Lithoclast ${ }^{\circledR}$ EMS, Nyon Suisse), montrant que le dernier était plus efficace. Dans leur série, ils montrèrent que la composition des calculs était en majorité faite de phosphate de calcium (14 Calculs) et pour un cas de cystine.

- Nahlieli, et al.[14-19] rapportèrent dans de multiples occasions entre 1994 et 2001, leur propre technique. L'évolution de leur endoscope interventionnel est clairement visible tout au long de ces années. En 1997, ils publièrent un premier protocole d'ablation. Les calculs étaient de préférence retirés soit par voie endoscopique soit à l'aide de forceps, ou de pince à panier ou encore par succion. Si le calcul ne pouvait être extirpé par l'un de ces moyens, alors il était fragmenté in situ à l'aide de pince à mors coupants. Plus tard, ils essayèrent la lithotripsie intra-corporelle par l'intermédiaire d'ondes de choc électro-hydraulique (Calcultrip ${ }^{\circledR}$, K. Storz, Allemagne). Si la sialographie pratiquée en pré-interventionnelle montrait un canal trop fin pour pouvoir utiliser l'une de ces méthodes, alors le calcul était retiré par voie chirurgicale.

$13 \%$ d'échecs furent notés par Nahlieli soit par sténose trop serrée du canal, soit par une trop grande angulation du canal excréteur des glandes salivaires, ou encore par des localisations aberrantes du calcul. Des cas d'ablation de lithiase chez les enfants furent rapportés en 2000 avec des techniques similaires.

- En 1996, Ottaviani et Capaccio[20] rapportèrent leur propre expérience de lithotripsie extra-corporelle par onde 
de chocs électromagnétiques (Minilith SL-1/K. Storz). 52 patients qui présentaient les lithiases salivaires avaient été récusés pour l'endoscopie et ont subi un traitement par lithotripsie extra-corporelle. $46 \%$ des patients ont évacué complètement leur calcul après leur traitement, $31 \%$ ont été laissés avec des fragments de moins de $2 \mathrm{~mm}$ et $23 \%$ avec des fragments supérieurs à $3 \mathrm{~mm}$. Dans le dernier groupe $1 / 3$ a dû subir une sialadenectomie.

- Marchal, et al.[21, 22] publièrent leur expérience sur la sialoendoscopie en 2001 et 2002. Ils trouvèrent que l'endoscope semi-rigide était meilleur que les endoscopes rigides. Les 25 patients traités par cette nouvelle méthode d'ablation ont eu $96 \%$ de taux de réussite. La taille moyenne des calculs était de $3,2 \mathrm{~mm}$. Deux protocoles étaient employés. Commençant avec un endoscope de petit calibre pour faire le diagnostic et le repérage de la lithiase, ils passaient ensuite un endoscope plus large avec canal opérateur introduisant ainsi une instrumentation endocanalaire type sondes à panier (Dormia ${ }^{\circledR} /$ K. Storz). Ils purent ainsi faire l'ablation de lithiases allant jusqu'à $4 \mathrm{~mm}$ de diamètre. Pour les calculs de plus gros diamètre, l'endoscopie diagnostique était suivie d'une lithotripsie intracorporelle sous endoscopie à l'aide d'ondes de chocs electrohydrauliques (Calcultrip ${ }^{\circledR}$ ) ou de fragmentation par laser type Homium (HL probe/Coherent, Inc., Santa Clara, CA, USA), pratiquée sous anesthésie générale. Le temps moyen pour faire le diagnostic et le traitement variait de 26 à 73 minutes suivant les cas. $45 \%$ des patients ont eu besoin de plusieurs interventions.

\section{patients et hypothèses étiologiques}

1973 patients qui présentaient une pathologie salivaire ont été traités par endoscopie et/ou lithotripsie extra-corporelle. 1205 endoscopies ont été réalisées entre 1988 et 2004. L'âge moyen des patients est de 44,5 ans, allant de 4 à 85 ans. 26 endoscopies avec ablation de calcul ont été réalisées sur des enfants de 4 à 13 ans. Il y eut 768 patients traités par lithotripsie extracorporelle pour fragmentation de leur lithiase entre 1995 et 2004. L'âge moyen de ces patients est 45,5 ans, allant de 6 à 85 ans. La division est égale dans les deux sexes pour chacun des traitements.
La formation des calculs salivaires était classiquement décrite comme une infection chronique des glandes entraînant une diminution de la sécrétion salivaire avec formation dans un acini d'une petite particule de mucus. Cette formation de mucus s'enrobant ensuite de phosphate de calcium ou d'autres sels minéraux et donnant une lithiase et un processus inflammatoire chronique.

En fait, il apparaît qu'à l'interrogatoire des patients, 309 ( $25 \%$ du total des glandes lithiasiques) présentaient des antécédents familiaux avec au moins un parent ou un collatéral ayant eu une lithiase salivaire. 
191 patients ayant moins de 10 ans présentaient déjà une lithiase voire deux pour certains. Lors des examens radiologiques et des diagnostics, aucun n'était porteur d'une infection chronique des glandes salivaires, type parotidite chronique ou sub-mandibulite.

118 avaient plus de 10 ans.

Nous avons retrouvé deux fratries présentant la même pathologie lithiasique.

La répartition familiale par patient se fait de la façon suivante :

- père atteint : 46 ,

- mère atteinte : 53 ,
- grands-parents atteints : 87,

- frère ou sœur : 2 ,

- oncle ou tante : 66 ,

- cousin ou cousine : 22 ,

- collatéraux éloignés : 33.

Dans ces conditions, il nous apparaît très nettement que les lithiases salivaires sont beaucoup plus d'origine génétique et non infectieuse et encore moins métabolique. Ceci corrobore le fait que l'atteinte soit à $98 \%$ sur une seule glande salivaire et non sur toutes les glandes salivaires principales.

\section{traitements non invasifs des lithiases}

- endoscopie

L'endoscopie des glandes salivaires a pu être réalisée grâce aux progrès de la technologie et à la fabrication de fibroscopes ultra-fins de moins un millimètre de diamètre.

Nous avons réalisé la première endoscopie au monde d'une glande parotide en décembre 1988. Depuis, nous en avons effectué 1205.

Cette étude a pour but de démontrer les possibilités diagnostique et thérapeutique de cette technique qui s'est avérée d'une grande fiabilité et a permis l'ablation de 697 lithiases par les voies naturelles.

\section{MATÉRIEL ET MÉTHODES}

Techniquement, 1045 endoscopies, de 1988 à 2001, ont été réalisées à l'aide d'endoscopes ultra-fins souples «Olympus ${ }^{\circledR} 》$ de $0,4,0,8$ et $1,1 \mathrm{~mm}$ (fig. 3 a à c), et 160, de 2001 à 2004, avec un endoscope semi-rigide Stor $z^{\odot}$ de 1,2 $\mathrm{mm}$ de diamètre (fig. 4).

Le protocole utilisé est toujours le même depuis treize ans. Nous pratiquons dans un premier temps le repérage de la lithiase. Pour cela, nous effectuons un cliché sans préparation, de face ou de profil, ou mieux, une tomographie panoramique des maxillaires, suivie d'un cliché intra-buccal occlusal pour les glandes submandibulaires et pour les calculs situés dans les canaux excréteurs. Une échographie des glandes salivaires est pratiquée, ceci ayant pour but de visualiser le parenchyme glandulaire et de savoir s'il existe une souffrance glandulaire ou une atrophie glandulaire due aux infections chroniques (fig. 1). Dans un dernier temps, nous pratiquons une sialographie avec simplement injection du canal jusqu'à l'obstacle pour obtenir le diamètre du canal excréteur et son degré de dilatation (fig. 2). 

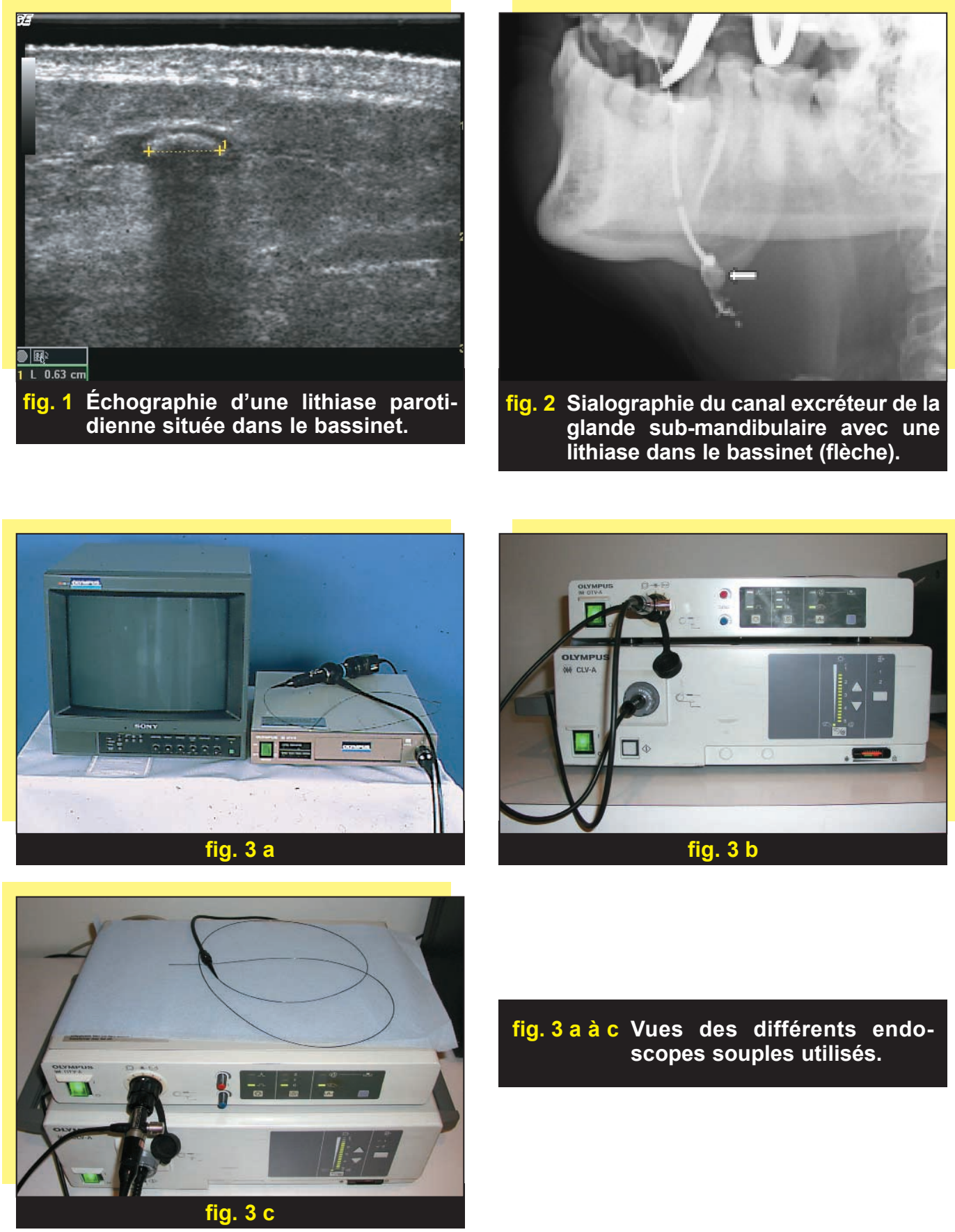

fig. 3 a à c Vues des différents endoscopes souples utilisés.

Si toutes les conditions sont réunies, c'est-à-dire un canal excréteur perméable, une lithiase mobile située dans ce canal et une glande salivaire non

atrophique et fonctionnelle, nous envisageons la fibroscopie.

L'introduction $\mathrm{du}$ fibroscope se fait après dilatation simple de l'ostium à 

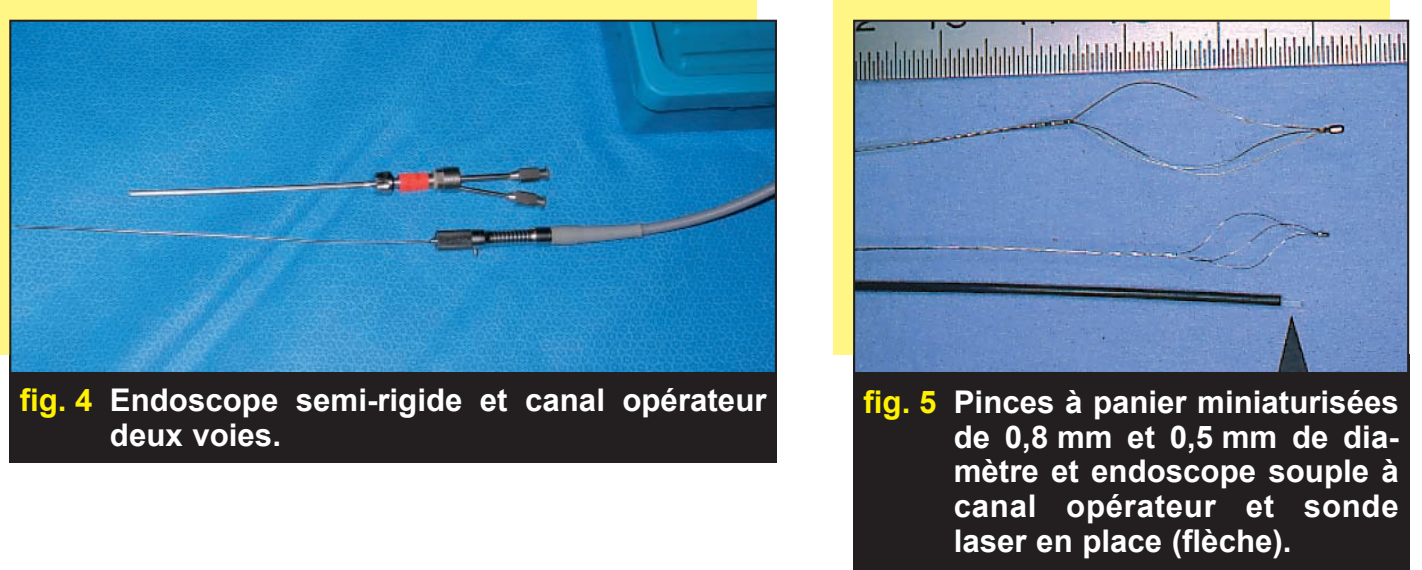

l'aide de sondes calibrées atraumatiques. Nous avons testé au cours de ces 13 années plusieurs diamètres d'endoscopes dont un de $1,1 \mathrm{~mm}$ avec canal opérateur de 500 microns permettant la mise en place d'une sonde laser à quartz de 300 microns. Ainsi, nous avons pu essayer de pratiquer des lithotripsies intra-canalaires à l'aide d'un Dye laser pulsé.

Devant l'extrême finesse du matériel endoscopique, il n'existe pas de béquillage et le guidage est effectué par des mouvements manuels par voie externe au contact de la peau ou de la muqueuse buccale.

Aucune anesthésie n'est nécessaire pour cet examen qui est totalement indolore.

Les ablations de calculs salivaires ont commencé en avril 1990, après réalisation d'une pince à panier miniaturisée de $0,8 \mathrm{~mm}$ de diamètre (Olympus ${ }^{\circledR}$ ). Cette dernière est descendue à l'aveugle dans le canal excréteur de la glande. En effet, il n'est pas possible de garder le fibroscope en place en raison de l'étroitesse des canaux salivaires (fig. 5).

\section{RÉSULTATS}

\section{Endoscopies}

À partir de décembre 1988 , nous avons examiné $66(6,4 \%)$ glandes salivaires réputées normales dont 34 (3,3\%) submandibulaires, $32(3,1 \%)$ parotides après sialographie, afin d'obtenir une anatomie endoscopique des différents canaux salivaires des glandes submandibulaires et parotides.

Ensuite, nous avons fibroscopé 1139 $(93,6 \%)$ glandes pathologiques, 921 $(72,5 \%)$ glandes lithiasiques, 618 $(50,1 \%)$ glandes submandibulaires, $303(22,4 \%)$ glandes parotides et 218 (21\%) glandes pathologiques présentant des infections chroniques (85 ou $7,8 \%$ ), des sténoses (64 ou $6,2 \%$ ), des ganglions (48 ou $5 \%$ ), des processus tumoraux (21 ou $2 \%)$ ). Au total, 1205 endoscopies ont été réalisées en 15 ans. L'examen a toujours été réalisé en ambulatoire sans arrêt de travail.

La répartition est équivalente dans les deux sexes, l'âge varie de 4 à 85 ans (moyenne 44,5 ans). 


\section{Ablations de lithiases}

Durant 13 ans, 697 lithiases ont été retirées par les voies naturelles, 526 (81\%) lithiases submandibulaires, 420 (58\%) étaient uniques, 106 (16\%) étaient multiples de 1 à 6 calculs, 177 (19\%) lithiases parotidiennes, 162 (16\%) uniques, 15 (1\%) multiples. Il a été possible de pratiquer 42 endoscopies chez des enfants âgés de 5 à 13 ans présentant des lithiases salivaires et d'en faire l'ablation sous endoscopie (35 lithiases submandibulaires et 7 lithiases parotidiennes), sous anesthésie locale de l'ostium du canal de Wharton ou de Sténon et avec des suites opératoires indolores (fig. 6 a à c).

En juillet 1992, nous avions commencé à pratiquer des lithotripsies in situ par
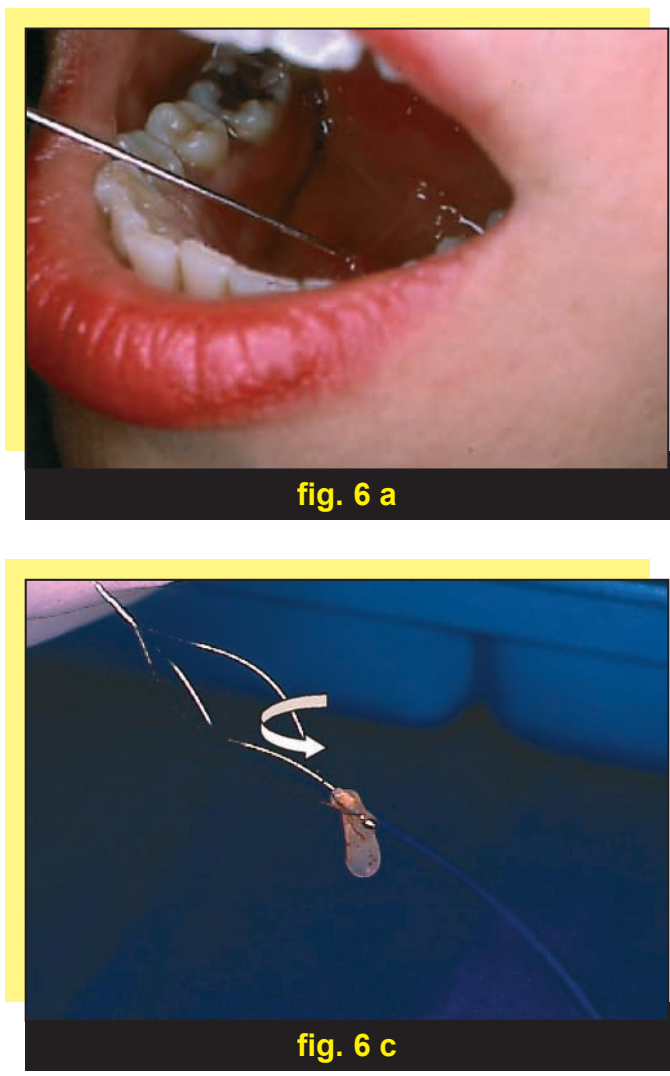

l'intermédiaire du laser pulsé à colorant (Pulsolith ${ }^{\circledR}$ Dye Laser Technomed International/France) sous contrôle endoscopique pour fragmenter les plus grosses lithiases bloquées soit dans le bassinet d'une glande, soit dans le canal excréteur, sans léser la glande ou le canal. En effet, l'action du laser pulsé à colorant n'est efficace que sur les lithiases de couleur jaune et de consistance dure et non pas sur les tissus mous de couleur rouge.

Nous avions totalisé entre juillet 1992 et mars 1994, 45 lithotripsies (39 calculs submandibulaires et 6 parotidiens). Les fragmentations étaient le plus souvent partielles permettant le rétablissement d'un flux salivaire, diminuant les crises de coliques salivaires et
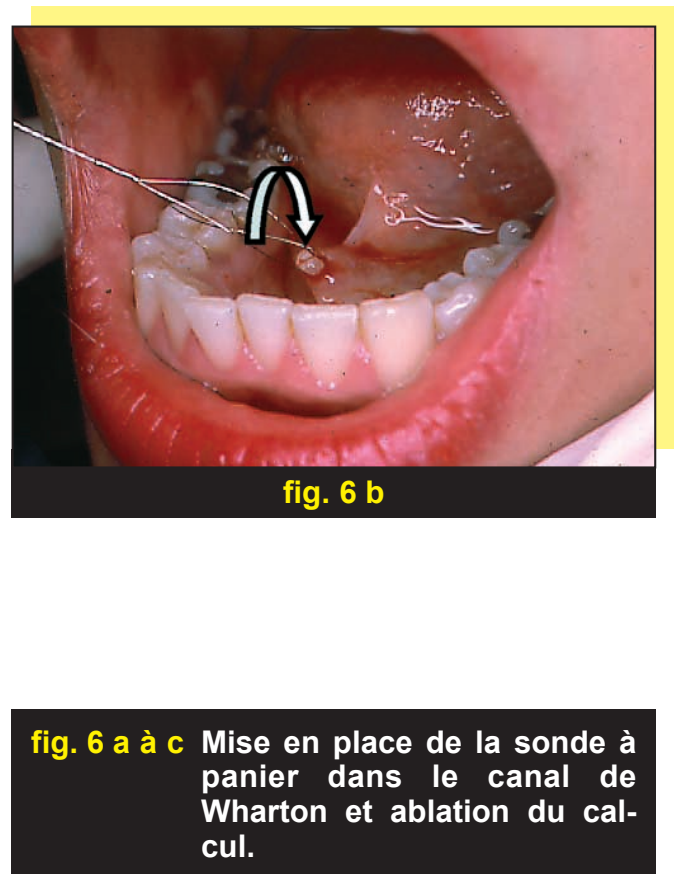
les infections. Plusieurs séances étaient nécessaires pour effectuer une fragmentation suffisante avec réduction de plus de $80 \%$ du volume du calcul. L'énergie délivrée par chaque impulsion laser était d'environ 40 à 60 millijoules à une fréquence de $10 \mathrm{~Hz}$. Une anesthésie locale était nécessaire pour pratiquer la fragmentation in situ. Ce procédé a été abandonné, en raison des contraintes mécaniques trop importantes imposées à l'endoscope endommageant la lentille frontale et remplacé par la lithotripsie extra-corporelle à ondes de choc électromagnétique.

Nous avons eu 53 échecs (3,1\%), 19 glandes submandibulaires présentaient des canaux de Wharton trop fins et 13 glandes parotides dont il a été impossible de franchir le coude du muscle buccinateur du canal de Sténon.

L'endoscopie a permis de mettre en évidence 102 (5\%) micro-lithiases radiotransparentes, non visibles par les moyens radiologiques conventionnels. Elle a aussi permis d'étayer le mécanisme physiopathologique des parotidites chroniques par absence d'une petite valvule normalement située à la jonction du canal de Sténon et du bassinet empêchant le reflux salivaire endo-parotidien.

\section{INCIDENT ET ACCIDENT DE L'ENDOSCOPIE}

Aucun incident ou accident n'est survenu jusqu'à ce jour au cours d'une endoscopie, mais il faut savoir qu'une dilatation mal conduite peut entraîner une perforation du canal excréteur de la glande, soit au niveau du muscle masséter pour la glande parotide, soit dans le plancher de la bouche pour la glande sous-maxillaire. Cet incident doit conduire à l'arrêt de l'examen.

L'endoscopie en elle-même doit être pratiquée de la façon la plus douce possible pour éviter toute perforation canalaire ou glandulaire. Le franchissement d'un obstacle doit être réalisé après avoir examiné les parois du canal, apprécié le degré d'inflammation à ce niveau et l'élasticité permettant le passage de l'endoscope. Toute manœuvre brusque doit être prohibée.

En ce qui concerne l'ablation de lithiase, nous avons eu un incident par blocage de la sonde à panier, après avoir clampé le calcul dans le bassinet de la glande parotide, au niveau de la jonction du canal de Sténon et de la glande. La sonde a été laissée en place et la parotidectomie superficielle avec conservation du nerf facial pratiquée quelque temps après.

Les extractions de lithiase salivaire ne doivent être pratiquées qu'après avoir attentivement examiné d'une part le calcul, d'autre part le canal excréteur, sa taille par rapport à la lithiase et son degré d'élasticité permettant le passage $\mathrm{du}$ corps étranger par les voies naturelles. En effet, une fois la lithiase attrapée dans la sonde à panier miniaturi-

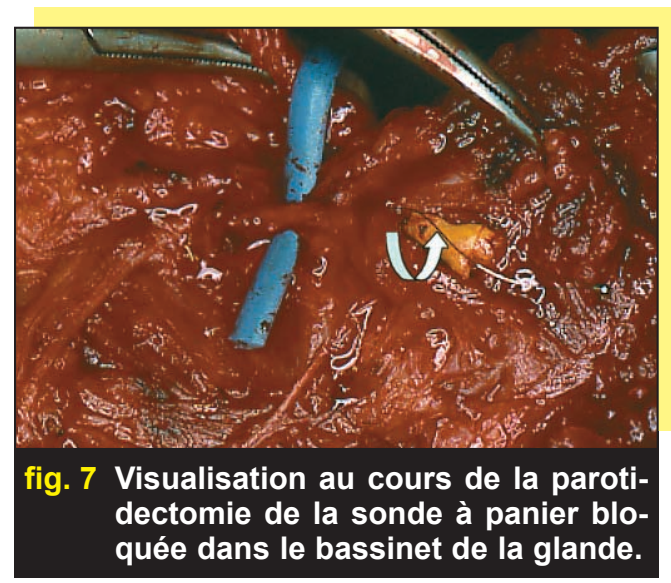


sée, il ne sera plus possible de la relâcher dans le canal, l'étroitesse des parois maintenant le calcul en place dans la sonde, et quelles que soient les manœuvres faites sur la sonde. Le risque majeur d'une extraction de lithiase salivaire mal conduite se traduira par l'ablation de la glande du fait du blocage de la sonde à panier miniaturisée, soit dans le bassinet, soit dans le canal principal de la glande.

Avec le développement de la lithotripsie extra-corporelle à ondes de chocs électro-magnétiques, ce type d'incident diminue, la fragmentation en fines particules de la lithiase permettant son élimination spontanée par l'ostium de la glande (fig. 7).

\section{- lithotripsie}

La lithotripsie extra-corporelle des calculs salivaires est désormais possible grâce à la fabrication d'un matériel miniaturisé utilisant les ondes de chocs électromagnétiques permettant la fragmentation sans lésions glandulaires.

\section{MATÉRIEL ET MÉTHODES}

Nous utilisons un nouvel appareil de lithotripsie extra-corporelle, le Minitlith SL1 ${ }^{\odot}$ (Storz Médical ${ }^{\odot}$ ) que nous avons mis au point et destiné uniquement aux glandes salivaires. Un repérage par sonde d'échographie de 7,5 MHz permet de situer exactement la lithiase et des ondes chocs électromagnétiques focalisées sur le calcul le fragmentent. Les résidus sont soit évacués spontanément par la salive dans le canal excréteur, soit enlevés par microvidéo endoscopique à l'aide d'une pince à panier miniaturisée. Ce nouveau traitement est pratiquement indolore, n'utilisant aucune anesthésie. Le patient est installé en position demiassise. L'onde de choc étant extrêmement focalisée, $2,5 \mathrm{~mm}$ sur $20 \mathrm{~mm}$. Les lésions sur les tissus avoisinants sont réduites. Son énergie est adaptée pour les calculs salivaires et varie de 5 à $30 \mathrm{mPa}$. La fréquence de tir est de 120 coups par minute, mais peut être réduite à 90 ou 60 coups par minute. Il

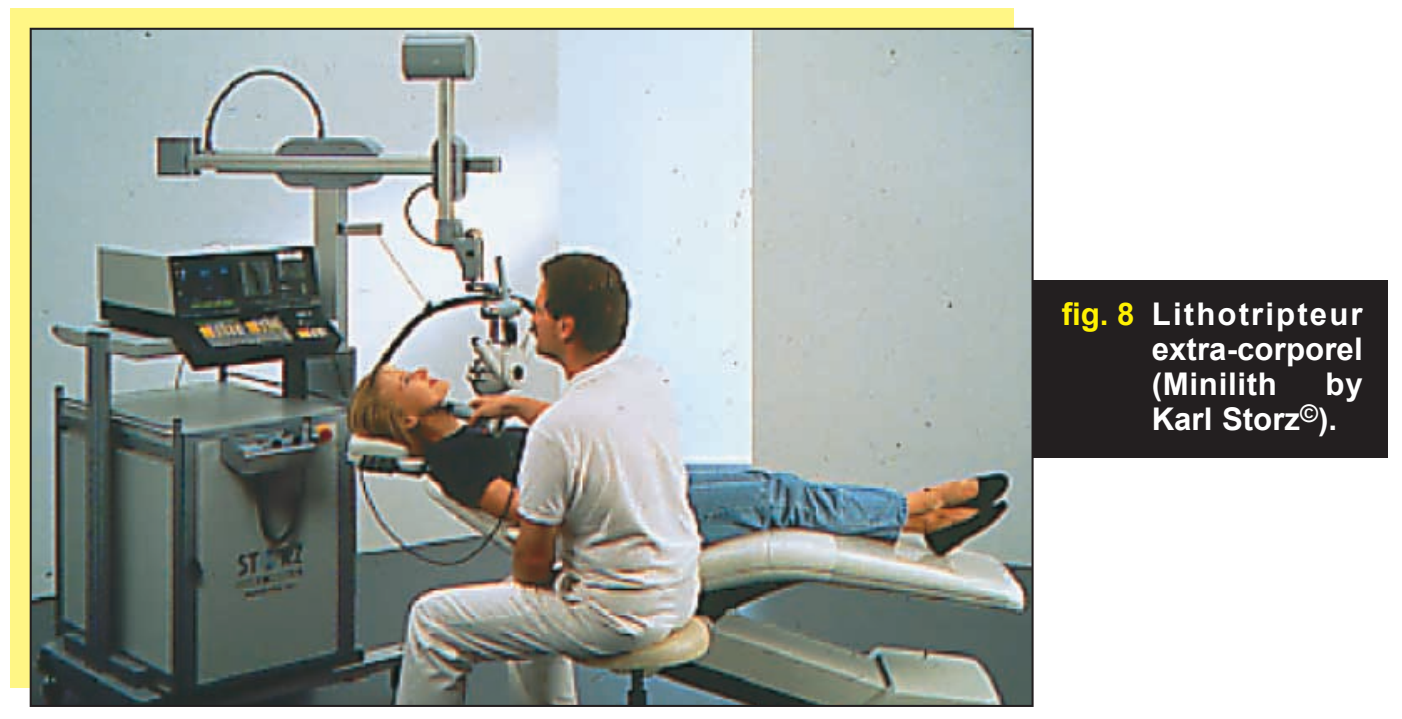


faut compter $1500+/-500$ coups par séance et chaque séance est espacée d'un mois (fig. 8).

\section{INDICATION ET CONTRE-INDICATION}

La lithotripsie peut être effectuée sur tous les calculs qui se trouvent dans les glandes submandibulaires ou parotides en excluant toutes lithiases dont l'abord pourrait être endoscopique ou chirurgical endobuccal, sans risque de lésion nerveuse tant au niveau du nerf lingual que du nerf facial. La taille de la lithiase n'a que peu d'importance, elle peut varier de $1 \mathrm{~mm}$ à $3 \mathrm{~cm}$, mais son repérage échographique doit être parfaitement effectué. Il ne doit exister aucune infection intra-glandulaire, au besoin un traitement anti-infectieux sera administré quelques jours avant la lithotripsie.

La sialographie reste l'examen radiologique de référence avant toute thérapeutique. En effet, il est le seul capable de donner le calibre du canal excréteur de la glande salivaire. Si ce canal est trop fin (moins d'un millimètre) pour laisser sortir les fragments lithiasiques, seul le traitement chirurgical restera valable.

La tomodensitométrie n'apporte que peu de renseignements sur le système canalaire glandulaire.

La résonance magnétique nucléaire n'est d'aucune utilité.

Enfin, il faut demander une crase sanguine. Toute perturbation de l'hémostase contre-indique la lithotritie. Le contrôle des traitements anti-coagulants doit se faire 24 heures avant la thérapeutique.

\section{RÉSULTATS}

De juillet 1995 à avril 2004, nous avons traité 768 patients, se répartissant en 377 (49\%) hommes et 391 (51\%) femmes.

L'âge des patients traités varie de 6 à 85 ans, soit une moyenne de 45,5 ans.

- 488 (65,5\%) présentaient une lithiase submandibulaire unique ou multiple, $280(34,5 \%)$ une lithiase parotidienne.

- 471 lithiases (63\%) ont été détruites (234 submandibulaires, 237 parotides). 272 (37\%) ont été réduites de volume avec des fragments résiduels de 3 à 5 mm (249 submanbibulaires, 23 parotides).

- 698 (97\%) patients ne présentent plus aucun symptôme douloureux de type colique salivaire au moment des repas.

Les fragments sont évacués spontanément dans la salive ou retirés à l'aide de la micro-pince à panier sous endoscopie avec sphinctérotomie de l'ostium du canal excréteur.

- 42 (3\%) patients ont continué de présenter des coliques salivaires, 25 $(2 \%)$ ont subi une submandibullectomie qui a permis de révéler un blocage des fragments à la jonction du canal de Wharton et bassinet avec un aspect macroscopique de sous-maxillite chronique, $12(1 \%)$ ont été mis sous traitement anti-infectieux, antiinflammatoires type corticoïdes et antalgiques et ont vu leurs signes régresser.

Nous pouvons remarquer que toutes les lithiases ont été fragmentées quelle que soit leur taille, soit $100 \%$ des cas traités (fig. 9 a et b, 10, 11). 

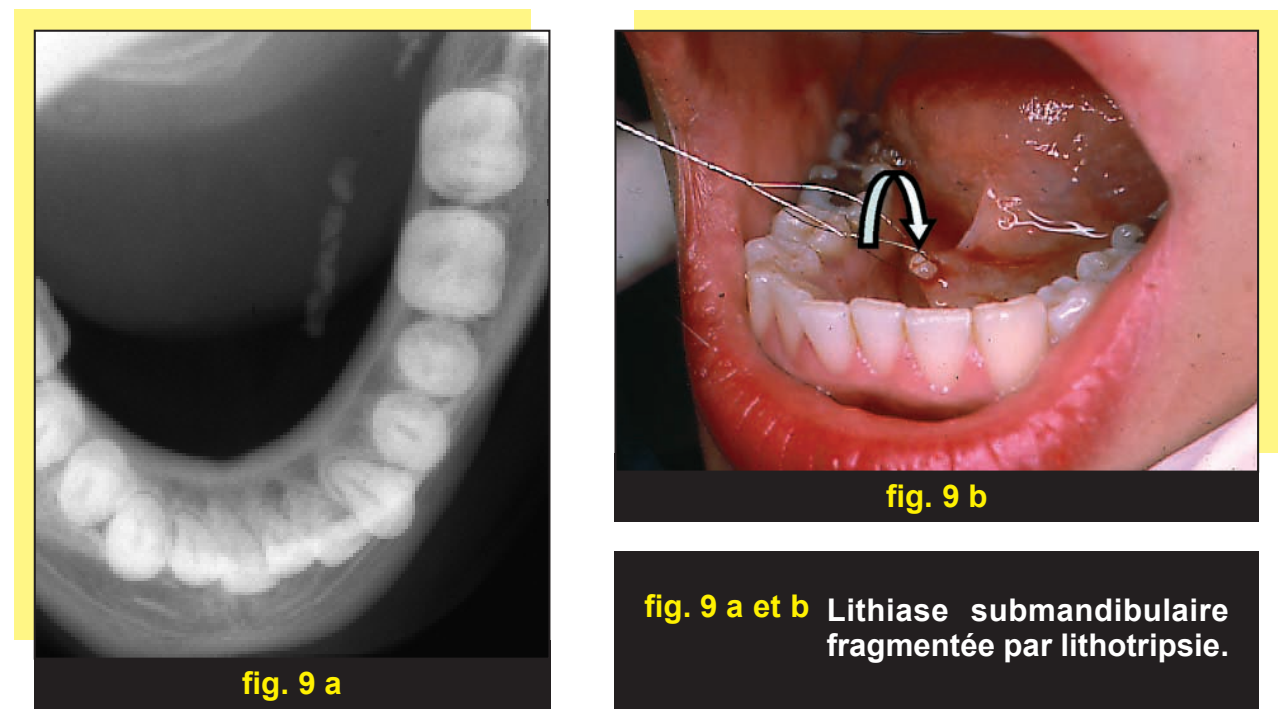

fig. 9 a et b Lithiase submandibulaire fragmentée par lithotripsie.
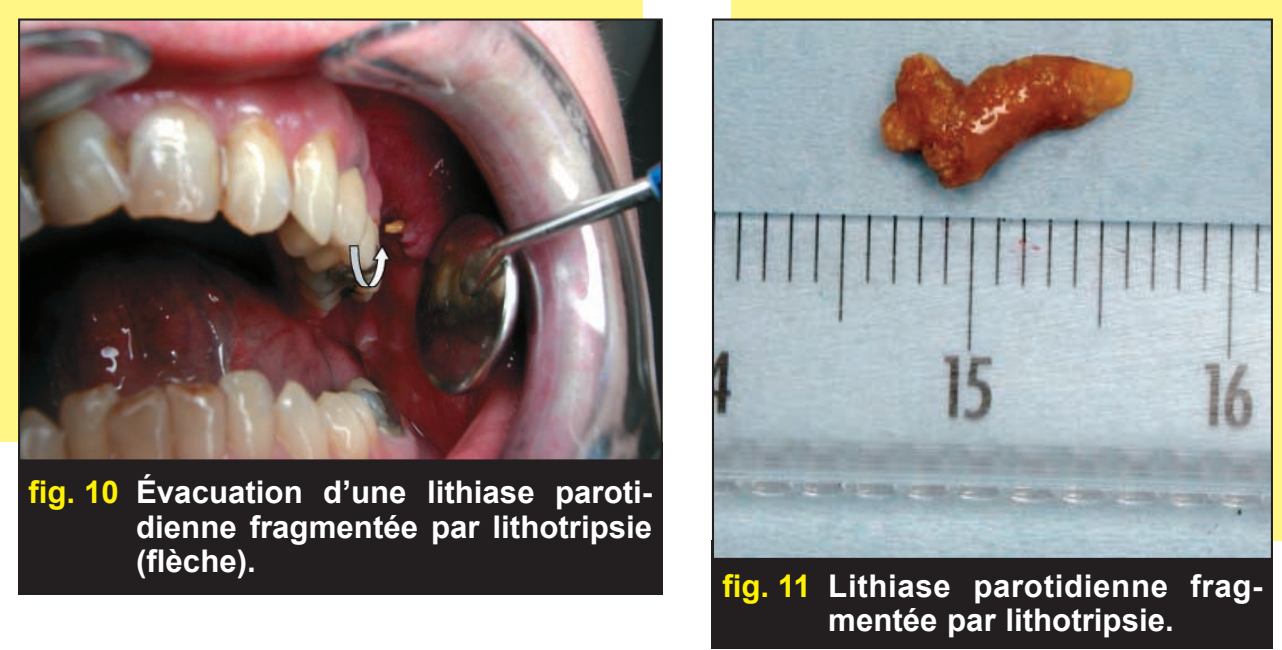

\section{EFFETS INDÉSIRABLES}

Quelques effets indésirables transitoires ont été observés au cours de la lithotripsie comme des pétéchies au point d'impact du tube (272 patients, $36 \%$ ), des douleurs légères s'associant à un gonflement de la glande traitée (180 patients, $22,5 \%$ ), des hémorragies canalaires (485 patients, $64,5 \%$ ) spontanément réduites.
Dans les 15 jours qui suivent le traitement, nous avons observé 310 (36\%) cas d'infections glandulaires traitées par antibiothérapie et corticoïde.

Notons qu'aucune anesthésie n'a été pratiquée, aucun sédatif majeur prescrit, et nous n'avons observé aucune lésion $\mathrm{du}$ nerf facial, transitoire ou définitive, au niveau de la glande parotide. 
Enfin, nous rappellerons la fragilité extrême du parenchyme glandulaire salivaire qui ne supporte pas les traumatismes et c'est la raison pour laquelle nous déconseillons toute tentative de lithotripsie sur des matériels non adap- tés comme les tables de lithotripsie rénale dont ni le repérage du calcul, ni la puissance de fragmentation, ni la position du patient ne sont adaptés aux glandes salivaires et entraîneraient des lésions irréversibles.

\section{discussion}

La phase de diagnostic doit permettre de mettre en évidence au niveau des glandes atteintes la meilleure méthode qui sera choisie pour l'ablation de la lithiase. Idéalement, un canal d'environ $3 \mathrm{~mm}$, une lithiase mobile et une glande non atrophique sont les meilleurs pronostics pour une endoscopie. Si ces conditions ne sont pas présentes avec un canal étroit, une lithiase enclavée et une glande peu fonctionnelle, alors l'endoscopie sera pratiquée de façon plus douce. L'endoscopie dépend aussi de l'anatomie des canaux excréteurs, en effet un coude du buccinateur trop accentué sur le canal de Sténon ou un canal de Wharthon trop fin ne permettront pas la mise en place de l'endoscope.

Normalement, aucune pathologie ne peut affecter la mise en place d'un endoscope ultra-fin de $0,8 \mathrm{~mm}$ de diamètre dans une glande salivaire. La présence de pus franc dans le canal excréteur d'une glande peut altérer la vision, mais un lavage doux à l'aide de sérum physiologique rétablira les conditions normales. L'endoscopie permet d'affiner certain diagnostic. En effet, des patients souffrant de coliques salivaires et dont les examens radiologiques se sont révélés normaux, ont pu, grâce à l'endoscopie voir leurs troubles mis en évidence. Des micro-lithiases radio-transparentes sont ainsi parfaitement détectées et traitées. L'efficacité de l'endoscopie est en général immédiate, entraînant après l'ablation du calcul une sédation des crises douloureuses. Il peut exister dans les 48 heures, une infection dûe au traumatisme du canalaire.

Un point important est à souligner, lorsqu'une glande salivaire a été mise en souffrance pendant une longue période par une lithiase, avec des infections chroniques, l'ablation de la lithiase entraîne une reprise de l'activité sécrétoire. Contrairement à ce qui était admis jusqu'à ce jour, les glandes salivaires ne sont que très rarement détruites complètement et leur activité peut reprendre lors de la levée de l'obstacle. Ceci a été parfaitement démontré sur des patients qui présentaient une atrophie glandulaire avant l'ablation du calcul et qui ont récupéré une fonction salivaire sub-normale après l'ablation de l'obstacle.

Les échecs de l'endoscopie ont été dus à des problèmes anatomiques, soit par la présence de canaux excréteurs trop fins, soit par des coudes du buccinateurs trop importants au niveau des glandes parotides. 
Les limites de l'ablation d'un calcul par voie endoscopique sont dues soit à la grosseur de la lithiase qui ne peut pas passer par le canal excréteur, soit à sa position, nichée dans le bassinet d'une glande ou enclavée dans un canal primaire ou secondaire.

Dans les premiers temps de la série, nous avons eu un accident par blocage de la pince à panier dans le bassinet d'une glande parotide lors de l'ablation d'une lithiase entraînant une parotidectomie. Nous avons alors pris les précautions nécessaires pour la poursuite de l'étude : tout calcul dépassant la taille de $3 \mathrm{~mm}$ et situé dans le bassinet d'une glande devait être soumis dans un premier temps à la fragmentation extracorporelle. C'est la raison pour laquelle nous n'avons que très peu d'incidents.

La composition chimique des calculs salivaires a été étudiée par quelques auteurs. La plupart des lithiases sont faites de plusieurs composants. Il est apparu pour certains auteurs $[6,13]$ que les calculs de couleur jaune étaient parfaitement détruits par le laser pulsé à colorant ou Dye laser Pulsolith ${ }^{\circledR}$; quoi qu'il en soit, la composition exacte n'a pas encore été déterminée.
La lithotripsie intra-corporelle a été commencée pour fragmenter les calculs de grandes tailles et ceux situés dans les bassinets des glandes. Fréquemment, cette fragmentation était partielle, permettant de rétablir temporairement le flux salivaire jusqu'à la session suivante, trois à quatre semaines plus tard. Les endoscopes souples étaient trop fragiles pour les contraintes physiques et cette méthode a été abandonnée au profit de la lithotripsie extracorporelle.

La lithotripsie extra-corporelle a pu être utilisée quelles que soient la taille et la position du calcul dans les glandes salivaires. Peu de contre-indications sont décrites pour cette méthode: infection aiguë, anomalie de la crase sanguine et prise d'anti-coagulants. Il est recommandé de n'utiliser que les lithotripteurs spécialement conçus pour les lithiases salivaires. Les lithotripteurs rénaux doivent être proscrits. Enfin, s'il apparaît que le canal excréteur de la glande est trop étroit, moins d'un millimètre de diamètre, ne pouvant laisser les fragments s'évacuer, seule l'intervention chirurgicale sera valable.

\section{conclusion}

La formidable évolution des nouvelles technologies non invasives a permis durant ces quinze dernières années de pratiquer une série de 1973 cas. L'endoscopie interventionnelle, avec la micro-instrumentation et la lithotripsie intra-corporelle, a permis de retirer avec succès les lithiases aussi bien dans les glandes sub-mandibulaires que paro- tides dans $96 \%$ des 1134 cas. La lithotripsie extra-corporelle pratiquée sur 768 cas a permis d'en détruire complètement $63 \%$ dont $35 \%$ ont été évacuées soit spontanément soit par endoscopie. Ceci a permis d'éviter aux patients une chirurgie plus lourde. Le diagnostic et la sélection doivent être correctement posés, afin d'utiliser la 
meilleure méthode soit endoscopie soit lithotripsie extra-corporelle. La chirurgie conventionnelle continue d'être effective et nécessaire dans les cas inappropriés et les échecs de ces nouvelles techniques.

\section{bibliographie}

1. Katz Ph, Fritsch $\mathrm{MH}$

Salivary stones: innovative techniques

in diagnosis

and treatment.

Curr Opin Otolaryngol

Head Neck Surg 2003;11: 173-178

2. Marmary $Y$.

A novel and non-invasive method for the removal of salivary gland stones. Int J Oral Maxillofac Surg 1986:15:585-587.

3. Brouns JJ, Hendrikx AJ, Bierkens AF.

Removal of salivary stones with the aid of a lithotripter. J Craniomaxillofac Surg 1989;17:329-330.

4. Katz Ph

Un nouveau mode d'exploration des glandes salivaires : la fibroscopie.

Information Dentaire 1990;8:785-786.

5. Katz Ph.

Endoscopie des glandes salivaires.

Ann Radiol (Paris) 1991 34(1):110-113.

6. Sterenborg $\mathrm{H}$

Van den Akker $\mathrm{H}_{\text {, }}$ Van der Meulen C, et al. Laserlithotripsy of salivary stones: a comparison between the pulsed dye laser and HO-YSGG laser. Med Sci 1990:5:357-362.
7. Grundlach P, Scherer $H$, Hopf J, et al.

Die endoscopisch

kontrollierte

laserlithotripsie

von speichelsteinen.

HNO 1990;38:247-250.

8. Konigsberger R, Feyh $\mathrm{J}$ Goetz A, et al.

Die ensoscopisch

kontrollierte

laserlithotripsie

zur behandlung

der sialolithiasis.

Laryngorhinootologie

1990;69:322-323.

9. Konigsberger R, Feyh J,

Goetz, et al.

Endoscopically-controlled

electrohydraulic

intracorporeal shock

wave lithotripsy (EISL)

of salivary stones.

J Otolaryngol 1993;22:

$$
\text { 12-13. }
$$

10. Kater W

Die fortentwicklung

des extrakorporalen

stoss wellen lithotripsie

von sperchelsteinen

mit dem minilith.

(Meeting)

65 jahresversammlung

des deutschen

gesellschaff fur orl

heilkumle kopf

and halschirugie.

Germany : Chemnitz, 1994(5).

11. Katz Ph

Traitement endoscopique des lithiasis salivaires.

ORL J (Fr) 1993; 42(1):

33-36.

12. Iro H, Zenk J.

Laser lithotripsy

of salivary duct stones.

Adv Otorhinolaryngol

1995:49:148-152.

13. Arzoz E, Santiago A,

Esnal $F$, et al.

Endoscopic

intracorporeal lithotripsy

for sialolithiasis.

J Oral Maxillofac Surg

1996:54:847-850.

14. Nahlieli $O$, Neder $A$, Baruchin AM.

Salivary gland endoscopy:

a new technique

for diagnosis

and treatment

of sialolithiasis.

J Oral Maxillofac Surg

1994;52:1240-1242.

15. Nahlieli O, Baruchin AM.

Sialoendoscope:

three years experience

as a diagnosis

and treatment modality.

J Oral Maxillofac Surg

1997; 55:912-918

16. Nahlieli O, Baruchin AM Endoscopic technique for the diagnosis and treatment of obstructive salivary gland diseases.

J Oral Maxillofac Surg 1999:57:1394-1401. 
17. Nahlieli $O$, Eliav $E$,

Hasson $O$, et al.

Pediatric Sialolithiasis.

Oral Surg Oral Med

Oral Path Oral Radiol

Endod 2000;90:709-712.

18. Nahlieli $O$, Baruchin AM.

Long-term experience

with endoscopic

diagnosis and treatment

of salivary gland

inflammatory

diseases.

Laryngoscope 2000:

988-993.
19. Nahlieli $O$, Shacham $R$, Yoffe B, et al.

Diagnosis and treatment of strictures and kinks in salivary gland ducts. J Oral Maxillofac Surg 2001;59:484-490

20. Ottaviani F, Capaccio $P$, Campi $\mathrm{M}$, et al.

Extracorporeal electromagnetic shock-wave lithotripsy for salivary gland stones. Laryngoscope 1996;106: 761-764.
21. Marchal $F$, Dulguerov $P$, Becker M, et al.

Specificity of parotid sialendoscopy. Laryngoscope 2001;111: 264-271.

22. Marchal $F$, Dulguerov $P$, Becker $\mathrm{M}$, et al. Submandibular diagnosis and interventional sialendoscopy: new procedure for ductal disorders. Ann Otol Rhinol Laryngol 2002;111:27-35.

\section{SUMMARY}

\section{Salivary lithiasis: alternative to surgical treatment}

P. KATZ

Objectives: salivary lithiasis is a relatively common medical problem. Treatment by invasive endoscopy, intracorporeal lithotripsy, and extracorporeal lithotripsy has been developing worldwide as an alternative to open surgical procedures.

We hypothesized that treatment of salivary gland duct stones with endoscopic ductal intervention and extracorporeal lithotripsy could eliminate ductal stones while exposing the patient to less morbidity. In the current study, we analyzed a large clinical series to determine the success of the new techniques and develop an algorithm for comprehensive treatment of salivary stones.

Study design: retrospective chart study of 1973 treated salivary ductal pathology cases.

Methods: treatment of salivary lithiasis using either duct endoscopy with instrumentation and/or intracorporeal laser lithotripsy or electromagnetic extracorporeal shock wave lithotripsy was undertaken for 1973 lithitic glands between 1988 and 2004. A total of 1205 endoscopies were performed on pathologic glands. 768 therapeutic extracorporeal lithotripsies were conducted.

keywords: salivary stones, endoscopy, lithotripsy.
Results: stones were eliminated by endoscopic technique in $96 \%$ of patients. Lithotriptic cases experienced stones completely destroyed in 63\% of cases, with an additional thirty-five percent of lithotriptic cases resulting in fragmentation of the stones with spontaneous expulsion or ancillary endoscopic removal. There were no major complications such as nerve or tooth damage. One patient early in the endoscopic series required surgical removal of a lodged wire-basket snare. Tissue effects such as edema, swelling, bleeding, or infection were temporarily present and treated as necessary. Four percent of endoscopic and 2\% of lithotriptor cases ultimately necessitated gland removal for stone removal.

Conclusions: the treatment algorithm of salivary stones which at one time contained only conventional open surgical means, may now include the use of endoscopes with instrumentation and extracorporeal lithotriptors. The two new techniques are especially valuable for stones located in the major ducts of the salivary glands. This series demonstrates the efficacies of both the endoscopic and lithotriptor techniques. 